\title{
Conserved Currents and Associated Symmetries; Goldstone's Theorem*
}

\author{
Daniel Kastler**, Derek W. Robinson**, and André SwiecA*** \\ University of Illinois, Urbana, Illinois
}

Received November 15, 1965

\begin{abstract}
We consider, in the framework of local field theory with translation symmetry, automorphisms connected with locally conserved currents. We show that such automorphisms lead to symmetries, i.e. are implementable by unitary operators, whenever the smallest mass in the theory is non-zero. Therefore we conclude that a "spontaneously broken symmetry" is possible only in the event that the smallest mass is zero. This establishes the theorem first conjectured by GoLDsTone.
\end{abstract}

\section{Introduction}

A great deal of attention has been recently focused upon the theory of symmetries and "broken symmetries" of physical theories, not only in the realm of elementary particles physics but also in the realm of solid state physics and the many-body problem. As the terms "symmetry" and "broken symmetry" have been used in various senses, let us immediately describe what we understand by this terminology.

All physical theories, such as quantum mechanics, field theory, etc. are at their most basic level algebraic and at this level all physical quantities described by the theory form a *-algebra $\mathfrak{A}$. In general there exist isomorphisms, or automorphisms, of the algebra i.e. mappings of the algebra into itself $\mathfrak{A} \rightarrow \mathfrak{A}$, and these automorphisms form the basis of the theory of symmetries. Although physical theories may be dealt with algebraically it is more usual to work at the representation level. Instead of considering the algebra $\mathfrak{A}$ a representation of $\mathfrak{A}$ by operators acting in a Hilbert space $\mathfrak{S}$ is considered and in all theories which are not purely statistical the extra proviso is normally made that the representation should be irreducible. This specialization to a representation introduces a distinction between the various possible kinds of automorphisms

* This work was supported by the National Science Foundation under contract NSF GP 2417.

** Present address: Physique Théorique, Faculté des Sciences, Univ. d'AixMarseille, France.

*** On leave of absence from University of Sao Paulo, Brazil. 
of $\mathfrak{A}$. Some of these automorphisms are implementable by unitary operators acting in the representation space $\mathfrak{F}$, and are thus called symmetries, whilst others are not implementable in this manner. By "spontaneous breakdown of symmetry" we shall mean exactly that this latter alternative occurs [1].

One reason why symmetries have been of interest in field theory is that in the Lagrangian formulation they lead not only to global conservation laws but also to laws of local current conservation. A recent problem has been to study to what extent the converse is true. Do local conservation laws lead to global symmetries? We wish to study this question in the framework of axiomatic field theory. There are essentially two problems posed by the question. The first problem is to characterize the conditions to be satisfied by the local current operators in order that there exist an automorphism of the basic algebraic elements defining the field theory. The second problem is then to find conditions under which the automorphism leads to a symmetry. We will focus our attention on this second problem.

We start off, in Section 2, by defining our field theoretic framework and the local conservation law. We assume that there is an automorphism of the $C^{*}$-algebra of quasilocal operations associated with the local current. We then consider, in Sections 3 and 4, under which conditions this automorphism leads to a symmetry. We prove that there is a symmetry if the smallest mass in the theory is non-zero. This result agrees with the theorem, first conjectured by GoLdstone [2], which states that if a local conservation law leads to a "broken symmetry" then the smallest mass in the theory must be zero. For an investigation of this problem which is similar in spirit to ours see Streater [3].

\section{Definitions and discussion}

We consider an algebraic field theory of the type formulated by HAAG and Kastler [4]. However, we are not able to work in a purely algebraic manner because we discuss the conserved current hypothesis which is only defined for operators in Hilbert space. Therefore we look at representations of the theory but we can, without loss of generality, consider only representations with special properties. Let us list the structural properties which are relevant.

1. Local structure: Corresponding to each open region $\mathcal{O}$ of spacetime ( $\mathcal{O}$ is supposed to be an open domain with compact closure) there is a $C^{*}$-algebra $\mathfrak{A}(\mathcal{O})$ of (strictly) local operations. The set theoretic union of all $\mathfrak{A}(\mathcal{O})$ is a normed *-algebra and its uniform closure is a $C *$. algebra $\mathfrak{A}$, the algebra of quasi-local operations. We consider a faithful irreducible representation of $\mathfrak{A}$ by bounded operators in a Hilbert 
space $\mathfrak{G}^{1}$. In principle all faithful representations contain the same physical information [4].

2. Local commutativity: If the regions $\mathcal{O}_{1}$ and $\mathcal{O}_{2}$ are totally spacelike to one another then

$$
\left[\mathfrak{U}\left(\mathcal{O}_{1}\right), \quad \mathfrak{A}\left(\mathcal{O}_{2}\right)\right]=0 .
$$

3. Translation symmetry: Corresponding to translations $y \rightarrow y+x$ in space-time there exist automorphisms $A \rightarrow A(x)$ of $\mathfrak{A}$ such that $\mathfrak{A}(\mathcal{O})$ goes into $\mathfrak{A}(\mathcal{O}+x)$. It is further assumed that in the representation under consideration the translations are implementable by unitary operators $T(x)$, i.e.

$$
A(x)=T(x) A T(x)^{-1} .
$$

4. Spectrum condition: The group of translation operators $T(x)$ has a spectral decomposition of the form

$$
T(x)=E_{0}+\int d E(p) e^{i p x} .
$$

The operator $E_{0}$ projects onto the vacuum state i.e. a state $\Omega$ which is translationally invariant

$$
T(x) \Omega=\Omega
$$

and $E(p)$ is a projection-valued measure which is assumed to have support only in $V_{m}^{+}$where

$$
V_{m}^{+}=\left\{p: p^{2} \geqq m^{2} \quad p_{0}>0\right\} .
$$

It is well known that if the representation of $\mathfrak{A}$ is irreducible then there is only one invariant state.

5. Local current conservation: There exist for every test-function $f(x)$ of class $\mathscr{D}$ four unbounded operators $j_{\mu}(f)$ with the properties

a) $\Omega$ is the domain of $j_{\mu}(f)$ and $\left(\Omega, j_{\mu}(f) \Omega\right)=0$.

b) $T(a) j_{\mu}(f) T^{-1}(a)=j_{\mu}\left(f_{a}\right)$ where $f_{a}(x)=f(x-a)$.

c) $\sum_{\mu=0}^{3} j_{\mu}\left(\frac{\partial f}{\partial x_{\mu}}\right)=0$

d) $\left(\Omega,\left[j_{\mu}(f), A\right] \Omega\right)=0$ for $A \in \mathscr{A}(\mathcal{O})$ if the support of $f(x)$ is totally space-like to $\mathcal{O}$. We take of course the natural definition

$$
\left(\Omega,\left[j_{\mu}(f), A\right] \Omega\right)=\left(j_{\mu}(f) \Omega, A \Omega\right)-\left(A^{*} \Omega, j_{\mu}(f) \Omega\right) .
$$

e) $\left(\Omega, j_{\mu}(x) j_{v}(y) \Omega\right)$ is a tempered distribution.

Assumptions 1.-5. are not sufficient to allow us to consider the problem of symmetries and must be supplemented by further conditions which may be of two types. Either we may assume further detailed properties of the unbounded operators $j_{\mu}(f)$ e.g. properties concerning the domain of $j_{\mu}(f)$, and then attempt to prove the existence of

1 This presupposes that the algebra is primitive. However, the irreducibility is not essential to our argument. It could be avoided if we assume instead that the current is associated with the algebra. 
the group of automorphisms which would be expected to arise from the local current hypothesis or we might assume the existence of the automorphisms and characterize them by certain expected properties. In either case the question then remains open as to whether or not there exists a symmetry. As this latter question is the one which mainly interests us at the moment we follow the more modest programme of assuming the existence of automorphisms characterized as follows:

6. Group of automorphisms: There is a one-parameter group of automorphisms $A \rightarrow A^{\tau}$ of the algebra $\mathfrak{U}$ strongly continuous with respect to $\tau$, and with the properties

a) If

then

$$
\mathcal{O}_{L}=\left\{x ;|\mathbf{x}|+\left|x^{0}\right|<L\right\}, \quad L>0
$$

and

$$
A_{L} \in \mathfrak{A}\left(\mathcal{O}_{L}\right) \text { implies } A_{L}^{\tau} \in \mathfrak{A}\left(\mathcal{O}_{L}\right)
$$

b) If

where

$$
f_{R}(\mathbf{x}) \in \mathscr{D}_{R} \text { and } f_{d}\left(x_{0}\right) \in \mathscr{D}_{d}
$$

$$
\begin{gathered}
\mathscr{D}_{R}=\left\{f_{R}(\mathbf{x}) ; \quad f_{R}(\mathbf{x}) \in \mathscr{D} \text { and } f_{R}(\mathbf{x})=\left\{\begin{array}{lll}
1 & \text { for } & |\mathbf{x}|<R \\
0 & \text { for } & |\mathbf{x}| \geqq R+r
\end{array}\right\}\right. \\
\mathscr{D}_{d}=\left\{f_{d}\left(x_{0}\right) ; f_{d}\left(x_{0}\right) \in \mathscr{D}, f_{d}\left(x_{0}\right)=0 \text { for }\left|x_{0}\right| \geqq d\right. \\
\text { and } \left.\int d x_{0} f_{d}\left(x_{0}\right)=1\right\}
\end{gathered}
$$

then

$$
\left.\frac{d}{d \tau}\left(\Omega, A^{\tau} \Omega\right)\right|_{\tau=0}=i \lim _{R \rightarrow \infty}\left(\Omega,\left[j_{0}\left(f_{R} f_{d}\right), A\right] \Omega\right)
$$

for all strictly local $A$.

In order that assumption $6 \mathrm{~b}$ ) should be reasonable we must show that the right hand side exists and is independent of the representative $f_{d}\left(x_{0}\right)$ of $\mathscr{D}_{d}$. However before we do this let us first discuss in heuristic terms the reasons behind the latter assumption.

Using the local current we would normally believe that we could define a local "charge" operator and that this would be formally given by

$$
Q_{V}(t)=\int_{V} d^{3} x j_{0}(\mathbf{x}, t),
$$

where the integration is over a finite volume. Then it should follow that such expressions as $C_{A}(t)$ defined by

$$
C_{A}(t)=\lim _{V \rightarrow \infty}\left[\int_{V} d^{3} x j_{0}(\mathbf{x}, t), A\right]
$$

exist, at least for strictly local $A$, because the commutator would be independent of $V$ when this volume is large enough. Further, because the current $j_{\mu}(x)$ is conserved it is to be expected that $C_{A}(t)$ is independent 
of time. Then for $A_{L} \in \mathfrak{A}\left(\mathcal{O}_{L}\right)$ we would have formally

$$
C_{A}(t)=C_{A}(0)=\left[\int_{|\mathbf{x}| \leqq L} d^{3} x j_{0}(\mathbf{x}, o), A_{L}\right] .
$$

In the same manner we would expect that the mapping

$$
A^{\tau}=\lim _{V \rightarrow \infty} \exp \left\{i \tau \int_{V} d^{3} x j_{0}(\mathbf{x}, t)\right\} A \exp \left\{-i \tau \int_{V} d^{3} x j_{0}(\mathbf{x}, t)\right\}
$$

should have some meaning and then for $A_{L} \in \mathfrak{A}\left(\mathcal{O}_{L}\right)$ we would argue as above that

$$
A_{L}^{\tau}=\exp \left\{i \tau \int_{|\mathbf{x}| \leqq L} d^{3} x j_{0}(\mathbf{x}, o)\right\} A_{L} \exp \left\{-i \tau \int_{|\mathbf{x}| \leqq L} d^{3} x j_{0}(\mathbf{x}, o)\right\} .
$$

On this heuristic level we then see that $A_{L}^{\tau} \in \mathfrak{A}\left(\mathcal{O}_{L}\right)$ and that

$$
\left.\frac{d}{d \tau}\left(\Omega, A_{L}^{\tau} \Omega\right)\right|_{r=0}=\lim _{V \rightarrow \infty}\left(\Omega,\left[\int_{V} d^{3} x j_{0}(\mathbf{x}, t), A_{L}\right] \Omega\right) .
$$

These are the counterparts of the assumptions contained in 6 . The added complications contained in $6 \mathrm{~b}$ ) are due to our having used the testfunction $f_{R}(\mathbf{x})$ to simulate the integration of $j_{0}(\mathbf{x}, t)$ over a finite volume and the test function $f_{d}(t)$ to smear out in the time component. As we have already mentioned, it would be interesting to find what conditions are necessary for $j_{\mu}(x)$ in order that (11) could be given more than a formal meaning but our assumption $6 \mathrm{~b}$ ) avoids this question. We wish now however to prove that as a result of assumptions $1-5$ the group of automorphisms characterized by 6 is always unitarily implementable in $\mathfrak{G}$. As preparation for the proof of this statement we first derive some properties of vacuum expectation values.

\section{Vacuum expectation values}

Let us first show the consistency of assumption $6 \mathrm{~b}$ ) by proving

Lemma I. The commutator $C_{L}\left(f_{R} f_{d}\right)$ defined by

$$
C_{L}\left(f_{R} f_{d}\right)=\left(\Omega,\left[j^{0}\left(f_{R} f_{d}\right), A_{L}\right] \Omega\right)
$$

is independent of the choice of the functions $f_{R}(\mathbf{x})$ and $f_{d}\left(x^{0}\right)$ within the classes $\mathscr{D}_{R}$ and $\mathscr{D}_{d}$ respectively and independent of the class $\mathscr{D}_{R}$ for $R>L+d$.

Proof: Let us consider test-functions $f_{d_{1}}\left(x^{0}\right)$ and $f_{d_{3}}\left(x^{0}\right)$ from the classes $\mathscr{D}_{d_{1}}$ and $\mathscr{D}_{d_{2}}$ respectively and take $d_{1} \geqq d_{2}$ and hence $\mathscr{D}_{d_{1}} \supseteqq \mathscr{D}_{d_{2}}$. Using these test functions we define $\hat{f}\left(x^{0}\right)$ by

and note that

$$
\hat{f}\left(x^{0}\right)=\int_{-\infty}^{x^{0}} d x^{\prime 0}\left\{f_{d_{1}}\left(x^{\prime 0}\right)-f_{d_{2}}\left(x^{\prime}\right)\right\}
$$

$$
\left\{\begin{array}{l}
\hat{f}\left(x^{0}\right)=0 \quad \text { for } \quad\left|x^{0}\right| \geqq d_{1} \\
\hat{f}\left(x^{0}\right) \in \mathscr{D}
\end{array}\right.
$$


and

$$
\frac{d}{d x^{0}} \hat{f}\left(x^{0}\right)=f_{d_{1}}\left(x^{0}\right)-f_{d_{2}}\left(x^{0}\right) .
$$

Now using assumption $5 \mathrm{~d}$ ) we have

But

$$
j^{0}\left(f_{R} \frac{d \hat{f}}{d x^{0}}\right)=j^{0}\left(f_{R} f_{d_{1}}\right)-j^{0}\left(f_{R} f_{d_{2}}\right)=\mathbf{j}\left(\nabla f_{R} \hat{f}\right) .
$$

$$
\nabla f_{R}(\mathbf{x}) \hat{f}\left(x^{0}\right)=0
$$

unless $R<|\mathbf{x}|<R+r$ and $\left|x^{0}\right|<d_{1}$ and thus

$$
\left(\Omega,\left[\mathbf{j}\left(\nabla f_{R} \hat{f}\right), A_{L}\right] \Omega\right)=0 \quad \text { for } \quad R \geqq L+d_{1}
$$

by local commutativity.

Combining (15) and (16) we have

$$
\left(\Omega,\left[j^{0}\left(f_{R} f_{d_{1}}\right), A_{L}\right] \Omega\right)=\left(\Omega,\left[j^{0}\left(f_{R} f_{d_{2}}\right), A_{L}\right] \Omega\right)
$$

for $R>L+d_{1}$.

This shows that $C_{L}\left(f_{R} f_{d}\right)$ is independent of $f_{d}\left(x^{0}\right)$ and the fact that it is independent of the particular $f_{R}(x)$ and class $\mathscr{D}_{R}$ follows directly from local commutativity.

As a result of the above Lemma we see that the right hand side of (7) is indeed well defined and independent of $f_{d}\left(x^{0}\right)$. Before we proceed to prove other properties of vacuum expectation values let us introduce subalgebras $\mathfrak{A}_{n}$ of $\mathfrak{A}(n \geqq 0)$ by the following definition

$$
\mathfrak{A}_{n}=\left\{A ; L^{n} \operatorname{Inf}_{A_{L} \in \mathfrak{A}\left(\mathcal{O}_{L}\right)}\left\|A-A_{L}\right\| \underset{L \rightarrow \infty}{\longrightarrow} 0\right\} .
$$

In this notation we of course have $\mathfrak{A}=\mathfrak{A}_{0}$. We are now in a position to prove

Lemma II. If $A \in \mathfrak{A}_{n}$ with $n \geqq 2$ then

$$
\lim _{R \rightarrow \infty}\left(\Omega,\left[j^{0}\left(f_{R} \frac{d^{s} f_{d}}{d x^{0 s}}\right), A\right] \Omega\right)=0
$$

for all $s \geqq 1$.

Proof: We will prove the lemma for $s=1$, the result for $s>1$ follows analogously. From the local conservation law

$$
\left(\Omega,\left[j^{0}\left(f_{R} \frac{d f_{d}}{d x^{0}}\right), A\right] \Omega\right)=\left(\Omega,\left[\mathrm{j}\left(\nabla f_{R} f_{d}\right), A\right] \Omega\right)
$$

and because $A \in \mathfrak{A}_{n}$ we may write

$$
A=A_{L}+\Delta A_{L}, \quad A_{L} \in \mathfrak{A}\left(\mathcal{O}_{L}\right)
$$

with the assurance that

$$
\lim _{L \rightarrow \infty} L^{n}\left\|\Delta A_{L}\right\|=0 .
$$


Let us choose $L=\frac{R-d}{2}$; we then have from local commutativity that

$$
\left(\Omega,\left[j^{0}\left(f_{R} \frac{d f_{d}}{d x^{0}}\right), A\right] \Omega\right)=\left(\Omega,\left[\mathbf{j}\left(\nabla f_{R} f_{d}\right), \Delta \frac{\left.A_{R-d}\right]}{2} \Omega\right) .\right.
$$

Hence

$$
\begin{gathered}
\lim _{R \rightarrow \infty}\left|\left(\Omega,\left[j^{0}\left(f_{R} \frac{d f_{d}}{d x^{0}}\right), A\right] \Omega\right)\right| \leqq 2 \lim _{R \rightarrow \infty}\left\|\mathbf{j}\left(\nabla f_{R} f_{d}\right) \Omega\right\| \cdot\left\|\Delta A_{\frac{R-d}{2} \|}\right\| \\
\leqq 2 c \lim _{R \rightarrow \infty}(R+r)^{2}\left\|\Delta A_{\frac{R-d}{2}}\right\|=0
\end{gathered}
$$

In the last estimate we have used the majorization

$$
\begin{gathered}
\left|\int d^{4} x d^{4} y f_{d}\left(x^{0}\right) \frac{\partial}{\partial x^{i}} f_{R}(\mathbf{x})\left(\Omega, \mathbf{j}^{i}(x) \mathbf{j}^{k}(y) \Omega\right) \frac{\partial}{\partial y^{k}} f_{R}(y) f_{d}\left(y^{0}\right)\right| \leqq \\
\leqq c^{2}(R+r)^{4} .
\end{gathered}
$$

To obtain this estimate we need only use the linked cluster property in its weakest sense [5] i.e.

Hence

$$
\left|\left(\Omega, j_{\alpha}(x) j_{\beta}(y) \Omega\right)\right| \underset{(x-y)^{2} \rightarrow-\infty}{\longrightarrow} 0 .
$$

$$
\left\|\mathbf{j}\left(\nabla f_{R} f_{d}\right) \Omega\right\| \leqq c_{1} R^{2} \text {. }
$$

This estimate, and hence the result of Lemma $I$, can be improved in a number of ways. Firstly, if we choose $r=R$ then $\nabla f_{R}(x) \sim 0\left(\frac{1}{R}\right)$ and if we further use the spectrum condition even with $m=0$ we have from the results of ARAKr, HEPP, and RUELLE [6] that

$$
(x-y)^{2}\left|\left(\Omega, j_{\alpha}(x) j_{\beta}(x) \Omega\right)\right| \leqq c_{2} \text { for }(x-y)^{2}<0,
$$

and this allows us to deduce

$$
\left\|\mathbf{j}\left(\nabla f_{R} f_{d}\right) \Omega\right\| \leqq c_{3} R .
$$

If we use the strong spectrum condition $m>0$ then RUELLE [7] has shown that

$$
\lim _{(x-y)^{2} \rightarrow-\infty}(x-y)^{2 N}\left|\left(\Omega, j_{\alpha}(x) j_{\beta}(y) \Omega\right)\right|=0 \text { for all } N>0
$$

and hence we deduce the optimal majorization

$$
\left\|\mathbf{j}\left(\nabla f_{R} f_{d}\right) \Omega\right\| \leqq c_{4} R^{1 / 2}
$$

Thus the strong spectrum condition is irrelevant in the proof of Lemma II but it is essential to prove

Lemma III. If $A \in \mathfrak{A}_{n}$ then there is an $A^{\prime} \in \mathfrak{A}_{n}$ such that, given $k \geqq 1$

and

$$
\{A-(\Omega, A \Omega)\} \Omega=\left(P^{0}\right)^{k} A^{\prime} \Omega
$$

$$
\left\{A^{*}-\left(\Omega, A^{*} \Omega\right)\right\} \Omega=\left(P^{0}\right)^{k} A^{\prime *} \Omega
$$


where $P^{0}$ is the energy operator (the infinitesimal generator of time translations).

Proof: We prove the lemma for $k>1$ as follows. We first define

$$
\bar{A}=A-(\Omega, A \Omega)
$$

and consider the spectral decomposition of the translations,

Since

$$
T(x)=E_{0}+\int e^{i p x} d E(p) .
$$

and

$$
E_{0}+\int d E(p)=I
$$

we have

$$
E_{0} \bar{A} \Omega=0
$$

this can be written

$$
\bar{A} \Omega=\int d E(p) \cdot \bar{A} \Omega
$$

$$
\bar{A} \Omega=\left(P^{0}\right)^{k} \int\left(p^{0}\right)^{-k} d E(p) \cdot \bar{A} \Omega
$$

and, taking account of the energy gap, i.e. the fact that the projectionvalued measure $d E(p)$ has its support contained in $V_{m}^{+}$, we next write

$$
\begin{aligned}
\bar{A} \Omega & =\left(P^{0}\right)^{k} \int \tilde{g}\left(p^{0}\right) d E(p) \cdot \bar{A} \Omega \\
& =\left(P^{0}\right)^{k} A(g) \Omega
\end{aligned}
$$

where we choose $\tilde{g}\left(p^{0}\right)$ to be a real even function of $p^{0}$ which is infinitely differentiable and such that $\tilde{g}\left(p^{0}\right)=\left|p^{0}\right|^{-k}$ for $\left|p^{0}\right| \geqq m$. The function $g\left(x^{0}\right)$, the Fourier transform of $\tilde{g}\left(p^{0}\right)$, is also real and even and we have used the notation

$$
\bar{A}(g)=\int d x^{0} g\left(x^{0}\right) A\left(x^{0}\right) .
$$

Let us take $A^{\prime}=\bar{A}(g)$ and we then notice that (38), (39), and (40) hold with $\bar{A}$ replaced by $\bar{A}^{*}=A^{*}-\left(\Omega, A^{*} \Omega\right)$ where $\bar{A}^{*}(g)=\bar{A}(g)^{*}$ owing to the fact that $g$ is real. To complete our proof we must finally show that $\bar{A}(g) \in \mathfrak{A}_{n}$ when $\bar{A} \in \mathfrak{A}_{n}$. For this we notice that because $\tilde{g}\left(p^{0}\right)$ is absolutely integrable and infinitely differentiable it follows that $g\left(x^{0}\right)$ is a continuous function decreasing rapidly at infinity. We can therefore find a decomposition of $g\left(x^{0}\right)$ for $L \geqq 0$ such that

$$
g\left(x^{0}\right)=g_{L}^{\prime}\left(x^{0}\right)+g_{L}^{\prime \prime}\left(x^{0}\right)
$$

where $g_{L}^{\prime}\left(x^{0}\right)$ is a continuous function which vanishes outside of the interval $[-L,+L]$ and where $g_{L}^{\prime \prime}\left(x^{0}\right)$ is a bounded continuous function with the property that

$$
L^{n}\left\|g_{L}^{\prime}\right\|_{1} \underset{L \rightarrow \infty}{\longrightarrow} 0
$$

where \|\|$_{1}$ denotes the $L_{1}$-norm:

$$
\|\varphi\|_{1}=\int\left|\varphi\left(x^{0}\right)\right| d x^{0} .
$$


Now as $\bar{A} \in \mathfrak{A}_{n}$ we may assume the decomposition $\bar{A}=\bar{A}_{L}+\Delta \bar{A}_{L}$ where

$$
\bar{A}_{L} \in \mathfrak{A}\left(\mathcal{O}_{L}\right) \text { and } L^{n}\left\|\Delta \bar{A}_{L}\right\| \underset{L \rightarrow \infty}{\longrightarrow} 0 .
$$

We finally have that $\bar{A}_{L}\left(g_{L}^{\prime}\right) \in \mathfrak{A}\left(\mathcal{O}_{4 L}\right)$ and

$$
\begin{aligned}
L^{n}\left\|\bar{A}(g)-\bar{A}_{L}\left(g_{L}^{\prime}\right)\right\| & \leqq L^{n}\left\|\bar{A}_{L}\left(g_{L}^{\prime \prime}\right)\right\|+L^{n}\left\|\Delta \bar{A}_{L}(g)\right\| \leqq \\
& \leqq L^{n}\left\|\bar{A}_{L}\right\| \cdot\left\|g_{L}^{\prime \prime}\right\|_{1}+L^{n}\left\|\Delta \bar{A}_{L}\right\| \cdot\|g\|_{1} \underset{L \rightarrow \infty}{\longrightarrow} 0
\end{aligned}
$$

as a consequence of (43) and (45) (notice that $\left\|\bar{A}_{L}\right\|$ is bounded since $\left.\left\|\bar{A}_{L}-\bar{A}\right\| \underset{L \rightarrow \infty}{\longrightarrow} 0\right)$.

The proof of the lemma for $k=1$ is slightly complicated by the fact that $\tilde{g}\left(p^{0}\right)$ is no longer integrable; however $g\left(x^{0}\right) d x^{0}$ is still a bounded measure (with a logarithmic singularity at the origin) ([8], p. 360, formula 34) and this is sufficient to construct a proof.

Now using Lemmas II and III we can prove the following Lemma which is the only result essential for the ensuing discussion of the symmetry associated with our group of automorphisms

Lemma IV. If $A \in \mathfrak{A}_{n}$ with $n \geqq 2$ then

$$
\lim _{R \rightarrow \infty}\left(\Omega,\left[j^{0}\left(f_{R} f_{d}\right), A\right] \Omega\right)=0 .
$$

Proof: As $\left(\Omega, j^{0}\left(f_{R} f_{d}\right) \Omega\right)=0$ we have that

$$
\left(\Omega,\left[j^{0}\left(f_{R} f_{d}\right), A\right] \Omega\right)=\left(\Omega,\left[j^{0}\left(f_{R} f_{d}\right), \bar{A}\right] \Omega\right)
$$

where, as above

$$
\bar{A}=A-(\Omega, A \Omega) .
$$

Now from Lemma III there is an $A^{\prime} \in \mathfrak{A}_{n}$ such that

and hence

$$
\bar{A} \Omega=\left(P^{0}\right)^{2} A^{\prime} \Omega \text { and } \bar{A}^{*} \Omega=\left(P^{0}\right)^{2} A^{*} \Omega
$$

$\left(\Omega,\left[j^{0}\left(f_{R} f_{d}\right), A\right] \Omega\right)=\left(\Omega, j^{0}\left(f_{R} f_{D}\right)\left(P^{0}\right)^{2} A^{\prime} \Omega\right)-\left(\Omega, A^{\prime}\left(P^{0}\right)^{2} j^{0}\left(f_{R} f_{d}\right) \Omega\right)$

$$
=-\left(\Omega,\left[j^{0}\left(f_{R} \frac{d^{2} f_{d}}{d x^{02}}\right), A^{\prime}\right] \Omega\right) .
$$

To obtain the last equality we have used the fact that

Thus

$$
P^{0} j^{0}\left(f_{R} f_{d}\right) \Omega=\left[P^{0}, j^{0}\left(f_{R} f_{d}\right)\right] \Omega=i j^{0}\left(f_{R} \frac{d f_{d}}{d x^{0}}\right) \Omega .
$$

$$
\lim _{R \rightarrow \infty}\left(\Omega,\left[j^{0}\left(f_{R} f_{d}\right), A\right] \Omega\right)=0
$$

as a result of (51) and Lemma II.

Lemma IV depends upon our assumption that the smallest mass $m$ is greater than zero because we needed the result of Lemma III to complete the proof. We note that under the same assumption, we can 
prove that for $A \in \mathfrak{A}_{n}$ with $n \geqq 2$

$$
\lim _{R \rightarrow \infty}\left(\Omega,\left\{j^{0}\left(f_{R} f_{d}\right), A\right\} \Omega\right)=0
$$

by using Lemma II and arguing as in the proof of Lemma IV ( \{\} denotes an anticommutator). Hence we can deduce that

$$
\lim _{R \rightarrow \infty}\left(\Omega, j^{0}\left(f_{R} f_{d}\right) A \Omega\right)=0 .
$$

As explained in the discussion following the proof of Lemma II these results can even be extended through use of the strong spectrum condition to all $A \in \mathfrak{A}_{1 / 2}$. This latter result is however optimal as may be inferred from discussion of the free charged field.

We have now prepared the ground for our discussion of symmetries which appears in the next section.

\section{Symmetry group}

The main result of the present work is now summarized by the following

Theorem. As a consequence of assumptions 1.-6. the automorphisms $A \rightarrow A^{\tau}$ are unitarily implementable. Specifically there exists a group of operators $U(\tau)$ such that

where

$$
A^{\tau}=U(\tau) A U(\tau)^{-1}
$$

and

$$
\begin{gathered}
U\left(\tau+\tau^{\prime}\right)=U(\tau) U\left(\tau^{\prime}\right) \\
U^{-1}(\tau)=U^{*}(\tau)=U(-\tau)
\end{gathered}
$$

Proof: Let us first note that if (56), (57), and (58) were valid then it would immediately follow that

$$
\left(\Omega, A^{\tau} \Omega\right)=(\Omega, A \Omega) .
$$

A fact that is perhaps less well known ${ }^{2}$ is that the converse is also true. Namely if (60) is true for all values of $\tau$ then there exist a group representation $\tau \rightarrow U(\tau)$ with the properties (56), (57), (58), and (59). This is an easy consequence of the Gelfand-Neumark-Segal $[10,11]$ construction. Let us recall that, if we denote abstract elements of $\mathfrak{A}$ by $a, b$ etc. and their respective representatives in the representation under consideration by $A, B$ etc. the latter are obtained in the following way: the Hilbert space $\mathfrak{G}$ is the completion of the quotient $\mathfrak{A} / \mathfrak{R}_{\omega}$, where $\mathfrak{R}_{\omega}$ is the null-ideal of the positive form $\omega$ associated with the cyclic state $\Omega$, i.e.

$$
\omega(a)=(\Omega, A \Omega)
$$

${ }^{2}$ However, see [9], Exercise 2.12.11. 
and

$$
\mathfrak{R}_{\omega}=\left\{a \in \mathfrak{U} ; \quad \omega\left(a^{*} a\right)=0\right\} .
$$

We will denote by $\hat{a}$ the class of $a \in \mathfrak{A}$ modulo $\mathfrak{R}_{\omega}$. We then have

and

$$
(A \Omega, B \Omega)=(\hat{a}, \hat{b})=\omega\left(a^{*} b\right)
$$

We start from (60) i.e.

$$
A \hat{b}=\hat{a b} \text {. }
$$

and define $U(\tau)$ by

$$
\omega\left(a^{\tau}\right)=\omega(a), \quad a \in \mathfrak{A}
$$

$$
U(\tau) \hat{a}=\hat{a^{\tau}} .
$$

$U(\tau)$ is thus coherently determined as a unitary operator on $\mathfrak{g}$ because

$$
(U(\tau) \hat{a}, U(\tau) \hat{b})=\omega\left(\left(a^{\tau}\right)^{*} b^{\tau}\right)=\omega\left(\left(a^{*} b\right)^{\tau}\right)=\omega\left(a^{*} b\right)=(\hat{a}, \hat{b}) .
$$

We have furthermore

$$
A^{\tau}=U(\tau) A U(\tau)^{-1}
$$

because using (66) it is immediately seen that the effect on $\hat{b}$ of both sides of $(68)$ is $\hat{a^{\tau}} b$. Finally $\tau \rightarrow U(\tau)$ is obviously a group representation which is strongly continuous due to our assumption that the one parameter group of automorphisms $a \rightarrow a^{\tau}$ is strongly continuous and the fact that $\omega$ is continuous (of norm unity), thus

$\|U(\tau) \hat{a}-\hat{a}\|=\omega\left(\left(a^{\tau}-a\right)^{*}\left(a^{\tau}-a\right)\right) \leqq\left\|\left(a^{\tau}-a\right)^{*}\left(a^{\tau}-a\right)\right\|=\left\|a^{\tau}-a\right\|^{2}$.

Notice that $U(\tau) \Omega=\Omega$ because $\Omega=\hat{e}$ and $e^{\tau}=e$, where $e$ is the unit element of $\mathcal{A}$.

The proof of our theorem is now reduced to proving $(60)$. It is sufficient to verify this for a strictly local $A$ because $|\omega(a)| \leqq\|a\|$ and every $a \in \mathfrak{A}$ is the limit in the norm topology of strictly local elements. Proving $(60)$ is equivalent to showing that, for all $\sigma$

But

$$
\left.\frac{d}{d \tau}\left(\Omega, A^{\tau} \Omega\right)\right|_{\tau=\sigma}=0 .
$$

$$
\left.\frac{d}{d \tau}\left(\Omega, A^{\tau} \Omega\right)\right|_{\tau=\sigma}=\left.\frac{d}{d \tau}\left(\Omega, A^{\tau+\sigma} \Omega\right)\right|_{i=0}=\left.\frac{d}{d \tau}\left(\Omega,\left(A^{\sigma}\right)^{\tau} \Omega\right)\right|_{\tau=0}=0 .
$$

The last expression vanishes according to assumption $6 \mathrm{~b}$ ), Lemma IV and the fact that $A^{\sigma}$ is strictly local, a consequence of assumption $6 \mathrm{a}$ ).

\section{Summary and Conclusions}

In the foregoing we have considered the problem of the existence of symmetries arising from automorphisms which are connected to locally conserved currents. We characterized the automorphisms by certain 
minimal assumptions which would be expected in all cases except for those automorphisms inherent in the space-time structure (translations, rotations and pure Lorentz transformations). Thus we would believe our characterization covers all automorphisms of the "internal" type e.g. gauge transformations of the first kind, isotopic spin, $S U(3)$ etc. In the framework of a field theory with local structure, translation invariance and a cyclic vacuum state we have shown that the automorphisms always lead to symmetries if the smallest mass in the theory is non-zero. Thus in the same framework we may conclude that if there is a "broken symmetry", the smallest mass must be zero. This conclusion confirms the conjecture of Goldstone.

Although we have followed Haag in formulating our field theory in an algebraic manner, our methods can also be applied to a Wightman type field theory. It might be stressed that although we have used an essentially relativistic framework we have nevertheless not needed to use the full Lorentz symmetry. Our results are independent of the existence of unitary operators implementing the homogeneous Lorentz group and consequently independent of the existence of covariant fields. The properties which are of major importance in the foregoing are the principle of locality, local commutativity and translation symmetry. These properties combined with the assumption that the smallest mass is non-zero imply that we have a theory of only short-range correlations and this does not allow a spontaneous breakdown of symmetry.

Let us conclude with a few remarks concerning further expected properties of the type of automorphism we have considered. These automorphisms should have the form

$$
A^{\tau}=\text { Norm } \lim _{R \rightarrow \infty} e^{i \tau j_{0}\left(f_{R} f_{d}\right)} A e^{-i \tau j_{0}\left(f_{R} f_{d}\right)}
$$

which should be compared to the heuristic form (10). We have left to a further paper the consideration of the properties of $j_{\mu}(f)$ which are necessary to derive (72). However, we note firstly that as a consequence of (72)

$$
A^{\tau}(x)=A(x)^{\tau} .
$$

Secondly we remark that although in the case of a symmetry

$$
\left(\Omega, A^{\tau} \Omega\right)=(\Omega A \Omega)
$$

it should not be inferred that the vector $\Omega_{R}$ defined by

$$
\Omega_{R}=e^{i \tau j_{0}\left(f_{R} f_{d}\right)} \Omega
$$

converges, in any sense, to the vector $\Omega$. Let us assume that it would converge weakly, then we would immediately infer that it converges strongly because

$$
\left\|\Omega_{R}\right\|=1 \text {. }
$$


However in this case we may use the inequality

$$
\left|\left(\Omega_{R}, A \Omega_{R}\right)-(\Omega A \Omega)\right| \leqq 2\left\|\Omega_{R}-\Omega\right\| \cdot\|A\|
$$

to infer that the left hand side tends to zero uniformly in $A$. This conclusion can easily be seen to be contradictory by choosing an $A$ localized in the region where $f_{R}(x)$ falls off to zero.

Acknowledgements. The major part of this work was accomplished whilst the authors were guests of the Physics Division of the Aspen Institute of Humanistic Studies whose hospitality and financial support by the National Science Foundation is gratefully acknowledged. The authors are indebted to Professor R. HAAG who provided a great deal of incentive to their work. Helpful discussions were also held with M. Guenin and H. Ezawa.

\section{References}

[1] HaAG, R.: Proc. of Boulder Meeting (1964); Borchers, H. J., R. HAAG, and B. Schroer: Nuovo Cimento 29, 148 (1963).

[2] Goldstone, J.: Nuovo Cimento 19, 154 (1961).

[3] Streater, R. F.: Phys. Rev. Letters 15, 475 (1965) and forthcoming manuscript.

[4] HAAG, R., and D. Kastler: J. Math. Phys. 5, 848 (1964).

[5] Jost, R., and K. Hepp: Helv. Phys. Acta 35, 34 (1962).

[6] Arakr, H., K. Hepp, and D. Ruelle: Helv. Phys. Acta 35, 164 (1962).

[7] Ruelle, D.: Helv. Phys. Acta 35, 147 (1962).

[8] Gelfand, I. M., and G. E. Shilov: Les Distributions. Paris: Dunod 1962.

[9] Dixmier, J.: Les $C^{*}$-Algebres et leurs représentations. Paris: GauthiersVillars 1964.

[10] Gelfand, I. M., and M. A. Neumark: Isvetija Ser. Math. 12, 445 (1948).

[11] Segal, I. E.: Bull. Am. Math. Soc. 53, 73 (1947). 